\title{
Novel insights into renal D-amino acid oxidase accumulation: propiverine changes DAAO localization and peroxisomal size in vivo
}

\author{
Lisanne Luks ${ }^{1} \cdot$ Silvia Sacchi $^{2,3} \cdot$ Loredano Pollegioni $^{2,3} \cdot$ Daniel R. Dietrich $^{1}$
}

\begin{abstract}
Chronic exposure to propiverine, a frequently prescribed pharmaceutical for treatment of overactive bladder and incontinence, provokes massive protein accumulation in the cytosol and nucleus of renal proximal tubule epithelial cells in rats. Previously, the accumulating protein was identified as D-amino acid oxidase (DAAO), a peroxisomal flavoenzyme expressed in kidney, liver and brain. The cellular mechanism of propiverine-induced DAAO accumulation, however, remains unexplained and poorly characterized. Therefore, to further increase the understanding of DAAO accumulation in rat kidney, this study aimed to characterize DAAO accumulations using differential immunofluorescent staining of rat kidney sections as well as in vitro binding analyses and proteasomal activity studies. We demonstrated that propiverine is neither a ligand of DAAO nor an inhibitor of the proteasome in vitro. However, propiverine treatment resulted in a significant decrease of peroxisomal size in rat proximal tubule epithelial cells. Moreover, peroxisomal catalase also accumulated in the cytosol and nuclei of propiverine-treated rats concurrently with DAAO. Taken together, our study indicates that
\end{abstract}

Daniel R. Dietrich

daniel.dietrich@uni-konstanz.de

1 Human and Environmental Toxicology, University of Konstanz, Universitätsstrasse 10, 78457 Constance, Germany

2 Department of Biotechnology and Life Sciences, University of Insubria, Varese, Italy

3 The Protein Factory Research Center, Politecnico di Milano and University of Insubria, Milan, Varese, Italy propiverine treatment affects the trafficking and/or degradation of peroxisomal proteins such as DAAO and catalase by a so far unique and unknown mechanism.

Keywords Propiverine $\cdot$ D-Amino acid oxidase $\cdot$ Renal protein accumulation $\cdot$ Protein mistrafficking
Abbreviations
Prop. Propiverine
rDAAO Rat D-amino acid oxidase
hDAAO Human D-amino acid oxidase
mDAAO Mouse D-amino acid oxidase
CD Circular dichroism
BF Bright field
ABCD3 ATP-binding cassette subfamily D member
LAMP2 Lysosomal-associated membrane protein 2

\section{Introduction}

In Europe and the USA, about $15 \%$ of adults suffer from overactive bladder (OAB) (Abrams and Andersson 2007). The International Continence Society defines symptoms of overactive bladder with urgency, urinary frequency, nocturia and urge incontinence (Wein 2011). Nowadays, increasing overweight and obesity are problematic since a positive correlation of weight and the extent of urinary incontinence has been reported (Subak et al. 2009). Propiverine is one of six anticholinergics which are recommended by the Committee on Pharmacological Treatment for the treatment of OAB (Madersbacher and Mürtz 2001). Upon oral administration to humans, propiverine is rapidly absorbed and undergoes extensive first-pass metabolism in the liver (Haustein and Huller 1988). The major hepatic metabolite formed is propiverine- $N$-oxide (Wuest et al. 2006) (Fig. 1). 


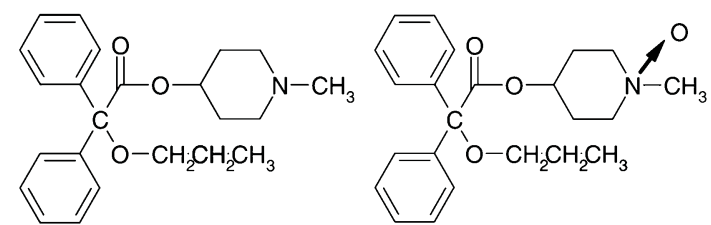

Fig. 1 Chemical structure of propiverine (left) and its major hepatic metabolite propiverine- $N$-oxide (right) (May et al. 2008)

Propiverine- $N$-oxide was also found to be the major metabolite formed in rats when administered orally, thus suggesting a similar pharmacological handling in humans and rats (Yamada et al. 2010). In order to attain drug approval for propiverine in Japan and later in the EU, preclinical testing was carried out in mice, rats and dogs (Inoue et al. 1989; Kohda et al. 1989; Nakano et al. 1989; Yamashita et al. 1989, 1990). In both the 13- and 52-week oral dosing study, rats presented with striking renal pathological changes: Propiverine treatment resulted in prominent protein accumulation in the cytosol and nuclei of proximal tubule epithelial cells (Nakano et al. 1989; Yamashita et al. 1990). These protein accumulations were more pronounced in males than in females, but did not result in overt cytotoxicity nor in secondary downstream pathological changes in the kidney (Yamashita et al. 1990). Contrary to the findings in rats, no such renal protein accumulation was observed in propiverine-exposed mice or dogs (Inoue et al. 1989; Kohda et al. 1989). Subsequently, the accumulated protein was isolated and characterized thereby suggesting the predominant accumulation of the protein D-amino acid oxidase (DAAO, EC 1.4.3.3) (Dietrich et al. 2008). DAAO is a FAD-dependent peroxisomal flavoenzyme that catalyzes the stereoselective oxidative deamination of D-amino acids to their $\alpha$-keto acids, hydrogen peroxide and ammonia (Pilone 2000; Sacchi et al. 2012). In peroxisomes, catalase subsequently ensures for the rapid elimination of the highly reactive hydrogen peroxide (Usuda et al. 1986; Pollegioni et al. 2007). Mammalian DAAO is expressed in kidney, liver and brain (descending order of expression level), whereby mice represent an exception as no hepatic expression was detected (Pollegioni et al. 2007; Konno et al. 2009). Rat DAAO (rDAAO) shares 93 and $80 \%$ sequence identity with mouse DAAO (mDAAO) and human (hDAAO), respectively (Konno 1998); nonetheless, rDAAO differs significantly from the human counterpart regarding structural, biochemical and kinetic properties: (1) rDAAO consists of 346 amino (38.8 kDa), whereas hDAAO consists of 347 amino acid residues $(39.5 \mathrm{kDa})$ (Konno 1998), (2) rDAAO is a monomer in the concentration range 1-20 mg/mL (Frattini et al. 2011) while hDAAO is a stable dimer (Molla et al. 2006), (3) rDAAO shows lower kinetic efficiency and a different substrate specificity as compared to the human counterpart (Frattini et al. 2011), and, most importantly, (4) rDAAO and hDAAO differ in affinity for the binding of classical inhibitors (Molla et al. 2006; Caldinelli et al. 2010; Frattini et al. 2011).

Intriguingly, strong evidence is provided that not only propiverine, but also a norepinephrine/serotonin reuptake inhibitor (NSRI) and other non-classified pharmaceuticals provoke intranuclear and -cytoplasmic DAAO accumulation in rat kidney (Gopinath et al. 1987; Hard et al. 1999; Radi et al. 2013). The above information on rDAAO characteristics and the finding that DAAO accumulation was exclusively observed in rat kidney upon treatment with propiverine or other compounds suggest that DAAO accumulation is a tissue- and species-specific phenomenon. Indeed, the latter assumption is also supported by the finding that spontaneous DAAO accumulation can be observed in a small percentage of Wistar Hannover rats (Shimoyama et al. 2014).

The DAAO accumulation in rat kidney appears similar to the well-described hyaline droplet nephropathy in male rats (Swenberg et al. 1989; Dietrich and Rasonyi 1995). This nephropathy is characterized by the renal proximal tubule epithelial accumulation of $\alpha 2$-urinary globulin, a fatty acid-binding protein synthesized exclusively in the liver of male rats and reabsorbed in the renal proximal tubules. The accumulation of $\alpha 2 \mathrm{u}$-globulin is governed by the reversible binding of compounds or their metabolites, resulting in slower enzymatic degradation of $\alpha 2 \mathrm{u}-$ globulin (Swenberg et al. 1989; Lehman-McKeeman et al. 1990; Frazier et al. 2012). Of note, the analogous mouse urinary protein (MUP) does not bind the $\alpha 2 \mathrm{u}$-binding compounds nor does it accumulate in the renal proximal tubules of mice (Dietrich and Rasonyi 1995), reminiscent of the situation described for the species-specific propiverine-induced rDAAO accumulation. Similar to rDAAO but more accentuated, $\alpha 2 \mathrm{u}$-globulin leads to renal protein overload already under normal physiological conditions (Read 1991; Shimoyama et al. 2014). However, while reduced enzymatic metabolism of $\alpha 2 \mathrm{u}$-globulin exacerbates only the accumulation in the lysosomes of the renal proximal tubules, exacerbated propiverine-induced rDAAO accumulation occurs in the cytosol and the nuclei of the renal proximal tubules (Cuervo et al. 1999; Dietrich et al. 2008). We thus raised the question whether compound interaction could affect rDAAO structure resulting in impaired protein degradation and/or targeting. Indeed, proteasomes are located both in the nuclei and the cytosol (Reits et al. 1997; Lee and Goldberg 1998; Wójcik and DeMartino 2003; von Mikecz 2006). Moreover, there is strong evidence that the subcellular localization of proteins is subject to significant alterations under particular stress conditions. Accordingly, during proteotoxic stress several proteins such as cell cycle-related proteins, 
transcription-coupled proteins and mitochondrial proteins but also chaperones are known to accumulate in nuclei upon proteasome inhibition (Iwata et al. 2009; Latonen 2011; Latonen et al. 2011). We thus investigated whether propiverine and its major metabolite could inhibit proteasomal activity and/or proper DAAO translocation into the peroxisomes. Consequently, we performed in vitro experiments with recombinant proteins to determine whether propiverine or propiverine- $N$-oxide acts as DAAO ligands and induces changes in DAAO structure/conformation. Applying immunofluorescence techniques to rat kidney sections of propiverine-treated animals, we specified the subcellular localization of rDAAO accumulations, investigated whether rDAAO accumulations affect peroxisomal morphology and whether accumulations are a potential resultant of abrogated protein trafficking.

\section{Materials and methods}

\section{Chemicals and reagents}

Unless otherwise stated, all chemicals were purchased form Sigma-Aldrich GmbH, Germany.

\section{Recombinant proteins}

Recombinant rDAAO and hDAAO were expressed in BL21(DE3)Star E. coli strain and purified as previously reported in (Frattini et al. 2011) and (Molla et al. 2006), respectively.

\section{Spectral analyses}

All spectroscopic experiments were performed at $15{ }^{\circ} \mathrm{C}$ in $20 \mathrm{mM}$ Tris- $\mathrm{HCl}$ (pH 8.0), $10 \%$ glycerol and 5 mM 2-mercaptoethanol. Ligand-binding experiments were performed by adding small volumes $(1-10 \mu \mathrm{L})$ of concentrated stock solutions of ligands to samples containing $1 \mathrm{~mL}$ of $10 \mu \mathrm{M}$ enzyme and monitoring the spectral changes between 250 and $800 \mathrm{~nm}$ (Harris et al. 1999). Near-UV CD spectra (250-350 nm) were recorded on a Jasco J-810 spectropolarimeter (cell path $=1 \mathrm{~cm}$ ) and analyzed using JASCO software (Jasco Europe, Italy) (Caldinelli et al. 2005).

\section{Experimental design of the in vivo study}

In brief, male and female F344 rats were exposed for 16 weeks to 0,300 or $1000 \mathrm{ppm}$ propiverine in the diet (fed ad libitum), upon which they were sacrificed and kidneys were taken for pathological assessment via formaldehyde fixation paraffin embedding (FFPE) with subsequent sectioning, as described in detail in (Dietrich et al. 2008).

\section{Immunohistochemistry}

Archived FFPE samples of dietary propiverine-exposed male F344 rats were cut to $2-3 \mu \mathrm{m}$ and mounted on glass slides. Sections were deparaffinized, rehydrated and microwaved for $20 \mathrm{~min}$ in Tris-EDTA buffer ( $\mathrm{pH} 9$ ). After blocking for $20 \mathrm{~min}$ in $1 \% \mathrm{BSA}+2 \% \mathrm{FCS}$, sections were incubated overnight at $4{ }^{\circ} \mathrm{C}$ with primary antibodies against DAAO (Santa Cruz, \#sc-26077) plus ABCD3 (Sigma, \#HPA032027), catalase (Santa Cruz, \#sc50508 (Fig. 4) or Life Technology, \#A21987 (Suppl. Fig. 1)), LAMP2 (Bioss, \#bs-2379R), a2u-globulin (Santa Cruz, \#sc374075) or 20S proteasome (Abcam, \#ac109525). Subsequently, sections were probed for $1 \mathrm{~h}$ at room temperature with AlexaFluor-conjugated secondary antibodies (Life Technology) and stained for 25 min with $0.5 \%$ Sudan black B to reduce autofluorescence (Sun et al. 2011). Sections were finally counterstained using Hoechst 33342 and mounted with fluorescence mounting medium (Dako, \#S3023). Control stainings (without primary and secondary antibodies, respectively) were performed to rule out the possibility of nonspecific reactivity of the antibodies.

\section{Confocal microscopy}

Immunostained kidney sections were analyzed with a point laser scanning confocal microscope (Zeiss, LSM 780 ), equipped with a $63 \times / 1.4$ oil objective and using the ImageJ software $(\mathrm{NIH}$, version 1.49). Mean intensities and counts were quantified in sections from a minimum of three different animals per dose group. For quantification of nuclear sizes, the maximum width (optimal plane using z-stack analysis) of the nuclei was verified and then measured using ImageJ. Similarly, the relative peroxisomal size was defined as the ratio of fluorescence area to number of particle counts per visual field. For counting particles, we adjusted the threshold using Otsu's algorithm, filled holes and applied a watershed-segmentation. At least three representative visual fields from each slide were randomly selected. Each visual field contained four to seven tubules.

\section{Proteasome activity assay}

A cell-free in vitro proteasome assay was used to test for impairment of the murine, human and rat $20 \mathrm{~S}$ constitutive proteasome by propiverine $(25 \mathrm{mM}$ stock) or its major metabolite propiverine- $N$-oxide ( $25 \mathrm{mM}$ stock). Murine and human constitutive proteasomes as well as the substrates Suc-Leu-Leu-Val-Tyr-AMC (AMC, $100 \mu \mathrm{M}$ ), Z-Leu-LeuGlu- $\beta$ NA $(\beta N A, 100 \mu \mathrm{M})$ for the chymotrypsin-like activity and the caspase-like activity of the proteasome were kindly provided by Dr. Sonja Erath, University of Konstanz. Rat constitutive proteasome was obtained from $R \& D$ 
Systems (E-352-050). Proteasome activity was assessed as previously described, with minor variations (Erath and Groettrup 2014). Briefly, $85 \mathrm{ng}$ of proteasome was incubated with $25 \mu \mathrm{L}$ of propiverine, propiverine- $N$-oxide or $4 \mu \mathrm{L}$ of MG132 (10 mM stock) for $30 \mathrm{~min}$ at $37{ }^{\circ} \mathrm{C}$. Subsequently, $25 \mu \mathrm{L}$ AMC or $\beta$ NA substrate was added and incubated for $60 \mathrm{~min}$ at $37{ }^{\circ} \mathrm{C}$. Finally, fluorescence was measured using a Tecan microplate reader with an optimal signal amplification. AMC was excited at $360 \mathrm{~nm}$ and emitted at $465 \mathrm{~nm}$, $\beta N A$ was excited at $340 \mathrm{~nm}$, and fluorescence was read at $411 \mathrm{~nm}$. The fluorescence of propiverine/propiverine- $N$-oxide and any of the other reagents was tested to exclude interference with fluorescent readings of the proteasome assay.

\section{Statistical data analysis}

All statistical analyses were performed using GraphPad Prism 5.03 software. Data were presented as mean \pm SEM. Statistical differences to the control were determined using a one-way ANOVA with a Dunnett's post-test.

\section{Results}

\section{Recombinant DAAO properties are unaffected by propiverine}

Propiverine provokes massive protein accumulation of D-amino acid oxidase (DAAO) in the cytosol and nucleus in renal proximal tubule cells of rats. The mechanisms underlying the observed process are so far poorly understood. However, a similar process, the accumulation of a2u-globulin, is a well-described phenomenon in rat kidney that is caused by the reversible binding of chemicals to $\alpha 2 \mathrm{u}$-globulin resulting in a decreased degradation of the protein (Swenberg et al. 1989). Thus, we first evaluated the ability of propiverine or propiverine- $N$-oxide to interact with recombinant rat DAAO (rDAAO). We performed spectral analyses using the recombinant rat and human (hDAAO) proteins, since it is well documented that the binding of ligands in the active site of the flavoenzyme yields to concentration-dependent perturbations of the visible absorbance spectrum (Harris et al. 1999; Molla et al. 2006; Frattini et al. 2011). Notably, only minimal changes in the spectrum were observed following the titration with both propiverine and propiverine- $N$-oxide, suggesting that neither of the two compounds is an rDAAO ligand (Fig. 2I, II). Note that minor changes in the differential spectrum for propiverine- $N$-oxide are not concentration-dependent (up to $1000 \mu \mathrm{M}$ was tested, data not shown) and therefore negligible (Fig. 2-II, inset). Since we cannot rule out that propiverine or propiverine- $N$-oxide interact with the protein in a different region with respect to the active site, we investigated whether the two compounds induce significant alterations of the rDAAO tertiary structure using circular dichroism (CD) spectroscopy analysis. Thus, rDAAO near-UV $\mathrm{CD}$ spectra at different concentrations of propiverine and propiverine- $N$-oxide (Fig. 2III, IV) were measured: The overall conformation of the flavoenzyme was unaffected by the titration with the two compounds, since no perturbation of the CD signal was detected. Correspondingly, all experiments were performed with hDAAO and provided for comparable results (data not shown). We thus assume that neither propiverine nor its major hepatic metabolite interacts with rDAAO or hDAAO and does not induce detectable changes in the protein tertiary structure.

\section{Propiverine induces loss of peroxisomal rDAAO localization and changes peroxisomal size}

To better characterize rDAAO accumulation, we visualized cytosolic and nuclear rDAAO in renal sections via fluorescence staining methodology (Fig. 3-I, A-C red channel). Whereas rDAAO demonstrated a punctuate distribution in control animals (Fig. 3-I, A), we observed a dose-dependent increase in rDAAO accumulation in nuclei and cytosol after propiverine treatment (Fig. 3-I, B and C). Using the peroxisomal marker ABCD3 (ATP-binding cassette subfamily $\mathrm{D}$, member 3) - a peroxisomal membrane proteinwe confirmed that rDAAO is located in the peroxisomes of control rats (Fig. 3-I, A, overlay). Propiverine treatment, however, induced a shift in rDAAO localization from the peroxisomes to the cytosol and the nucleus, resulting in a complete loss of peroxisomal rDAAO (Fig. 3-I, B and C, overlay). Nuclear accumulations of rDAAO were identified in every nucleus of affected renal proximal tubules (Fig. 3I, B and C; Fig. 3-II). In contrast, the number of cytosolic rDAAO accumulations appeared to increase dose-dependently (Fig. 3-I, B and C). Intranuclear rDAAO inclusions were surrounded by a halo suggestive of chromatin displacement (Fig. 3-II). Consequently, we quantified the size of nuclei with or without rDAAO accumulation and demonstrated that intranuclear rDAAO inclusions did not affect nuclear sizes (Fig. 3-III). Realizing that propiverine treatment resulted in a subcellular redistribution of $\mathrm{rDAAO}$, we asked whether lack of rDAAO integration into peroxisomes would affect peroxisomal size. Indeed, we observed a significant propiverine-dose-independent decrease in peroxisomal size (Fig. 3-IV). Peroxisomes in tubules containing rDAAO accumulations showed about a twofold decrease in size compared to peroxisomes in control animals (Fig. 3, IV). Taken together, propiverine not only induced a translocation of rDAAO into nuclei and a dose-dependent accumulation in the cytosol, but also a dose-independent reduction of peroxisomal size, reminiscent of an all or nothing effect. 
(I)

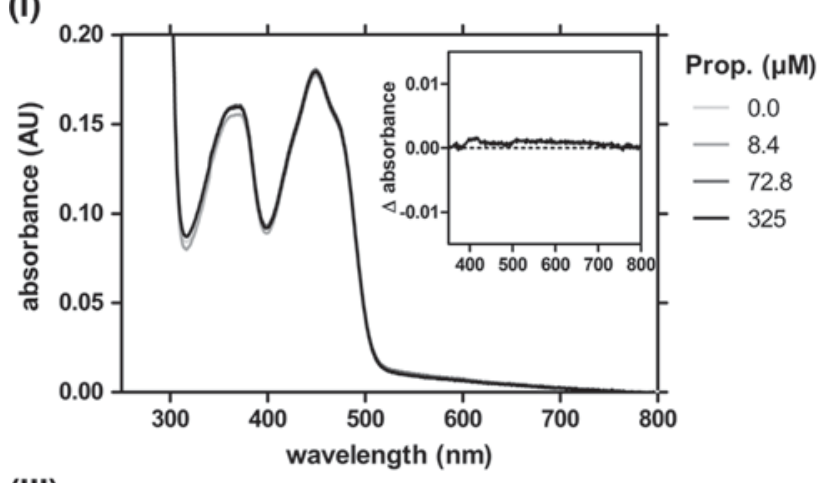

(III)

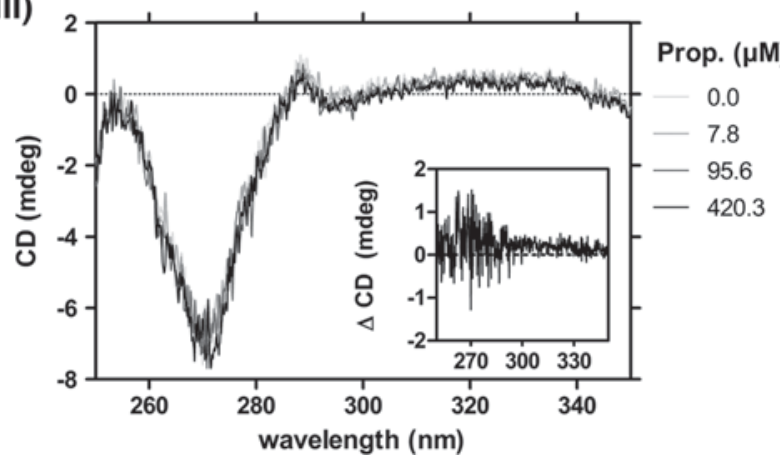

Fig. 2 Analysis of the binding of propiverine to rDAAO. I, II Absorbance spectra of recombinant rDAAO titrated with increasing concentrations of I propiverine and II propiverine- $N$-oxide. III, IV Near-UV circular dichroism of recombinant rDAAO titrated with

\section{Propiverine-induced mislocalization of peroxisomal catalase}

In view of the propiverine-mediated translocation of peroxisomal rDAAO, we asked whether the subcellular distribution of other peroxisomal proteins (e.g., catalase) is also affected. Accordingly, we co-stained rat kidney sections for rDAAO and catalase (Fig. 4). In control animals, catalase staining revealed a punctuate distribution which partially colocalized with rDAAO (Fig. 4A, yellow overlay). Using confirmatory immunofluorescence staining with antibodies to the peroxisomal membrane protein $\mathrm{ABCD} 3$, we were able to demonstrate that indeed both catalase and rDAAO were colocalized in renal proximal tubule peroxisomes of control animals (Suppl. Fig. 1A). Noteworthy, in propiverine-treated rats, although catalase was still partly present in peroxisomes (Suppl. Fig. 1B), it still largely colocalized with rDAAO in cytosolic and nuclear accumulations (Fig. 4B, overlay). This suggested a partial translocation of catalase from peroxisomes or abrogated peroxisomal import from the cytosol, similar to that observed for rDAAO. In summary, propiverine treatment resulted in the mislocalization of two peroxisomal proteins, DAAO and catalase, in rat renal proximal tubule cells.
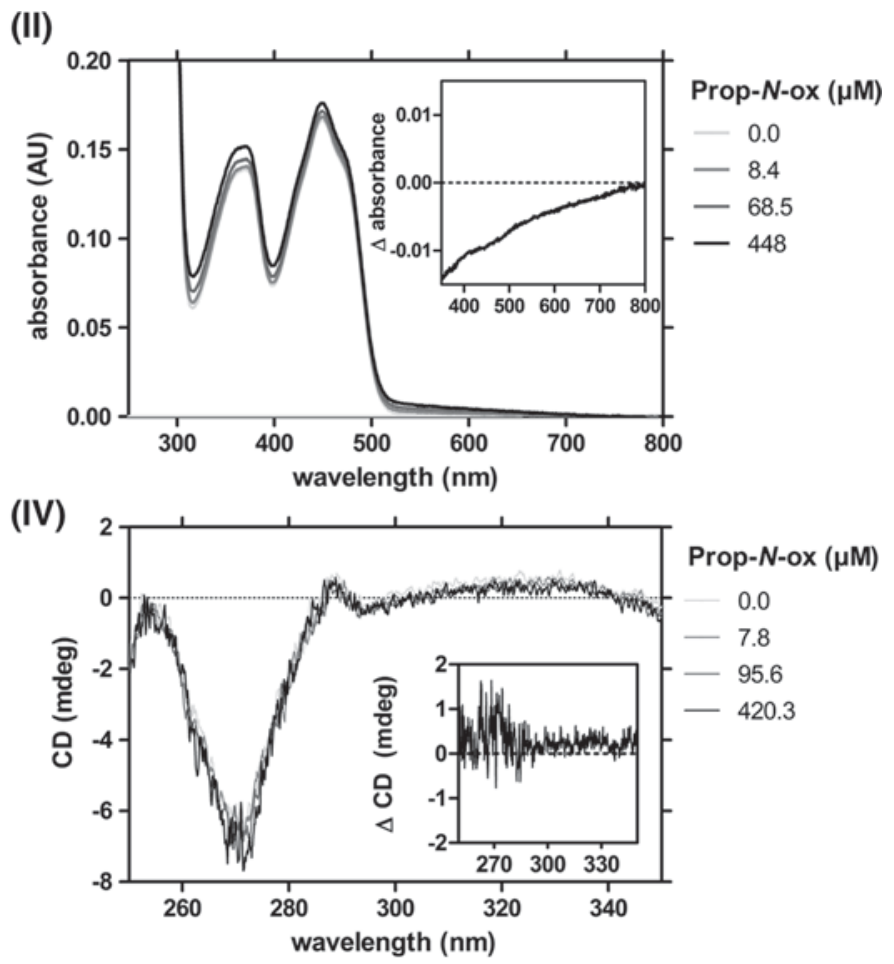

increasing concentrations of III propiverine and IV propiverine$\mathrm{N}$-oxide. Insets differential spectra obtained by subtracting the initial spectrum of the protein from the spectrum of the protein with the highest concentration of propiverine/- $N$-oxide

\section{Effects of propiverine on degradation pathways}

Having found that propiverine treatment induced the mislocalization of rDAAO and catalase, we asked whether these proteins are subject to impaired degradation, thereby resulting in a protein overload. In view of the fact that proteins can be degraded via the lysosomal-endosomal or the proteasomal pathway, we first analyzed whether cytosolic rDAAO accumulations are located in renal proximal tubule lysosomes. Therefore, rat kidney sections were stained for both rDAAO and the lysosomal-associated membrane protein 2, LAMP2 (Fig. 5-I). LAMP2 staining demonstrated punctuate staining in control and propiverine-treated animals (Fig. 5-I). However, while in control animals rDAAO was partly localized in lysosomes (Fig. 5-I, A), the rDAAO accumulations observed in propiverine-treated rats did not colocalize with, nor were they surrounded by LAMP2 (Fig. 5-I, B). As an additional control, we co-stained with an $\alpha 2 u$-globulin antibody (Fig. 5-II). As denoted earlier, $\alpha 2 \mathrm{u}$-globulin is a prominent male rat urinary protein which is nearly exclusively degraded in renal lysosomes (Read 1991). As expected, $\alpha 2 u$-globulin accumulations were highly prominent in the cytosol of both control and propiverine-treated male rats (Fig. 5-II). Of importance, however, was the observation that rDAAO did not colocalize with 
(I)

DAAO
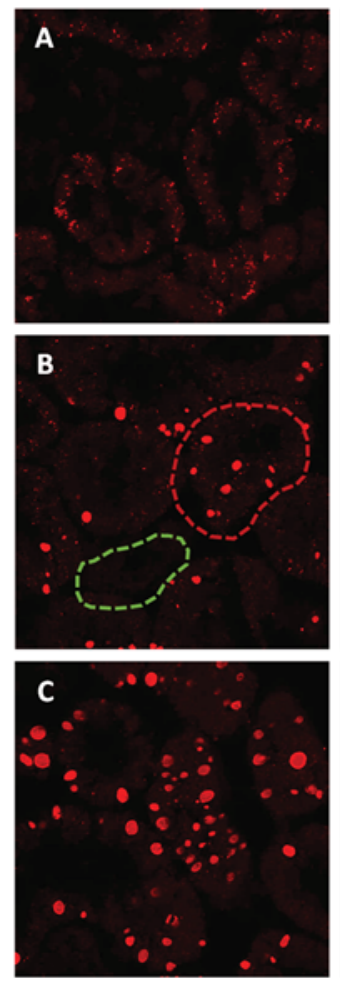

(II)
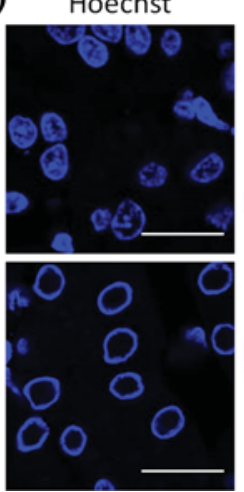

DAAO + Hoechst
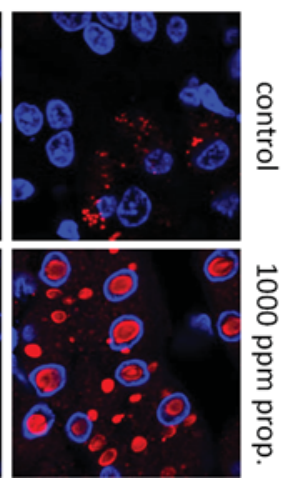

$\mathrm{ABCD} 3$
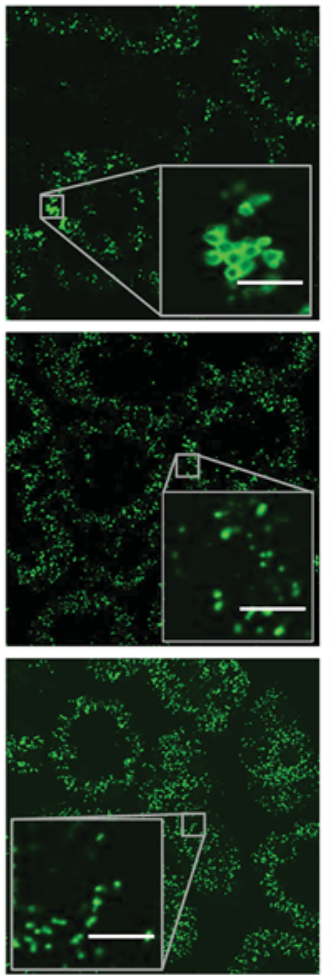

(III)
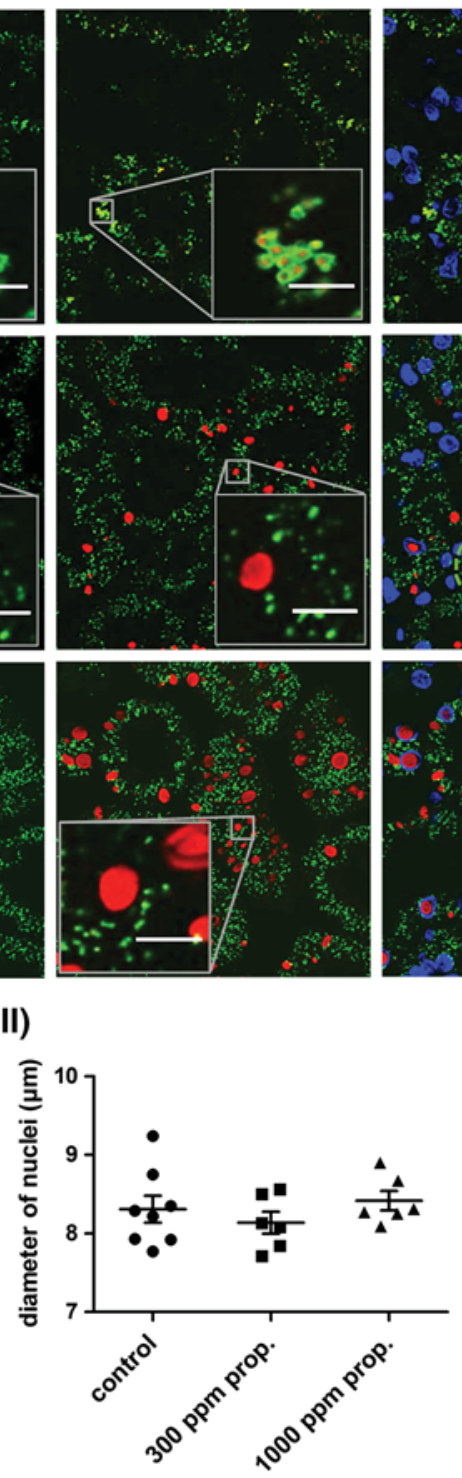

+ Hoechst
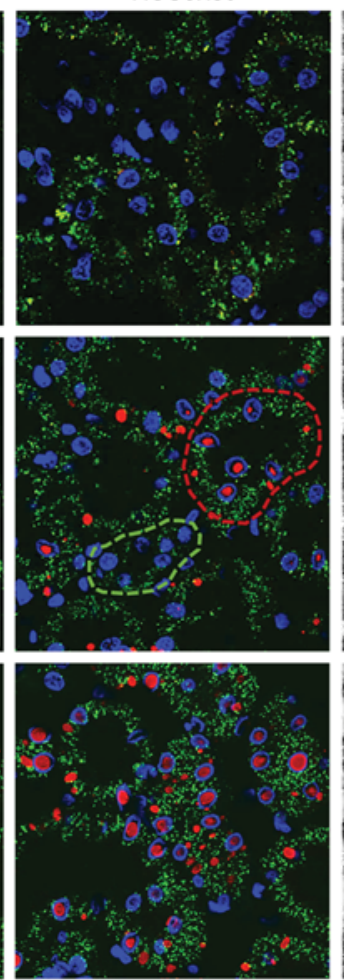

(IV)
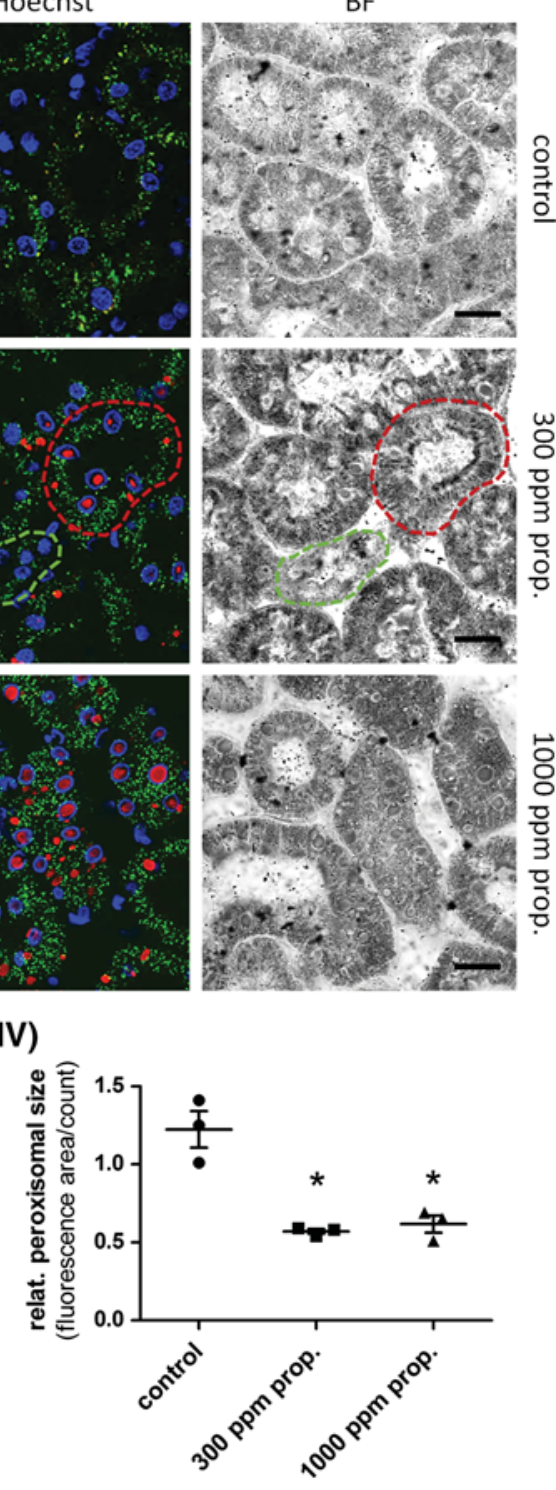

Fig. 3 Propiverine-induced changes in rDAAO localization and peroxisomal size. I Confocal microscopy of kidney sections of control and propiverine-treated male F344 rats. Co-staining of rDAAO and peroxisomal membrane protein ABCD3. Green delineation: unaffected distal tubule, red delineation: affected proximal tubule with rDAAO accumulation. Black scale bar $20 \mu \mathrm{m}$, scale bar in magnification box: $5 \mu \mathrm{m}$. II Co-staining of rDAAO and nuclei using Hoe-

$\alpha 2 \mathrm{u}$-globulin, thus confirming that rDAAO is not located in lysosomes (Fig. 5-II, B). More importantly, we did not observe translocation of $\alpha 2 \mathrm{u}$-globulin into the cytosol or the nuclei in propiverine-treated rats, suggesting that propiverine neither affected targeting of $\alpha 2 \mathrm{u}$-globulin, nor the uptake of $\alpha 2 \mathrm{u}$-globulin into the lysosomes (Fig. 5-II, B). chst 33342 in control and propiverine-treated rats. Scale bar $20 \mu \mathrm{m}$. III Nuclear diameter of Hoechst-stained proximal tubule cells. Dots indicate mean \pm SEM of 200 nuclei per animal; $n \geq 6$. IV Relative peroxisomal size in control- and propiverine-treated animals. Dots indicate mean \pm SEM of $15-25$ tubules per animal; $n=3$. One-way ANOVA with Dunnett's post-test. $* p<0.05 . B F$ bright field, $A B C D 3$ ATP-binding cassette subfamily D member 3 , prop. propiverine

As above data indicated that rDAAO accumulations are not located within lysosomes, we determined whether the proteasomal degradation pathway could be impacted by propiverine treatment. For this, we analyzed proteasomes and rDAAO in renal sections of control and propiverinetreated rats (Fig. 5-III). This immunostaining revealed that the majority of $20 \mathrm{~S}$ proteasomes are located within the 

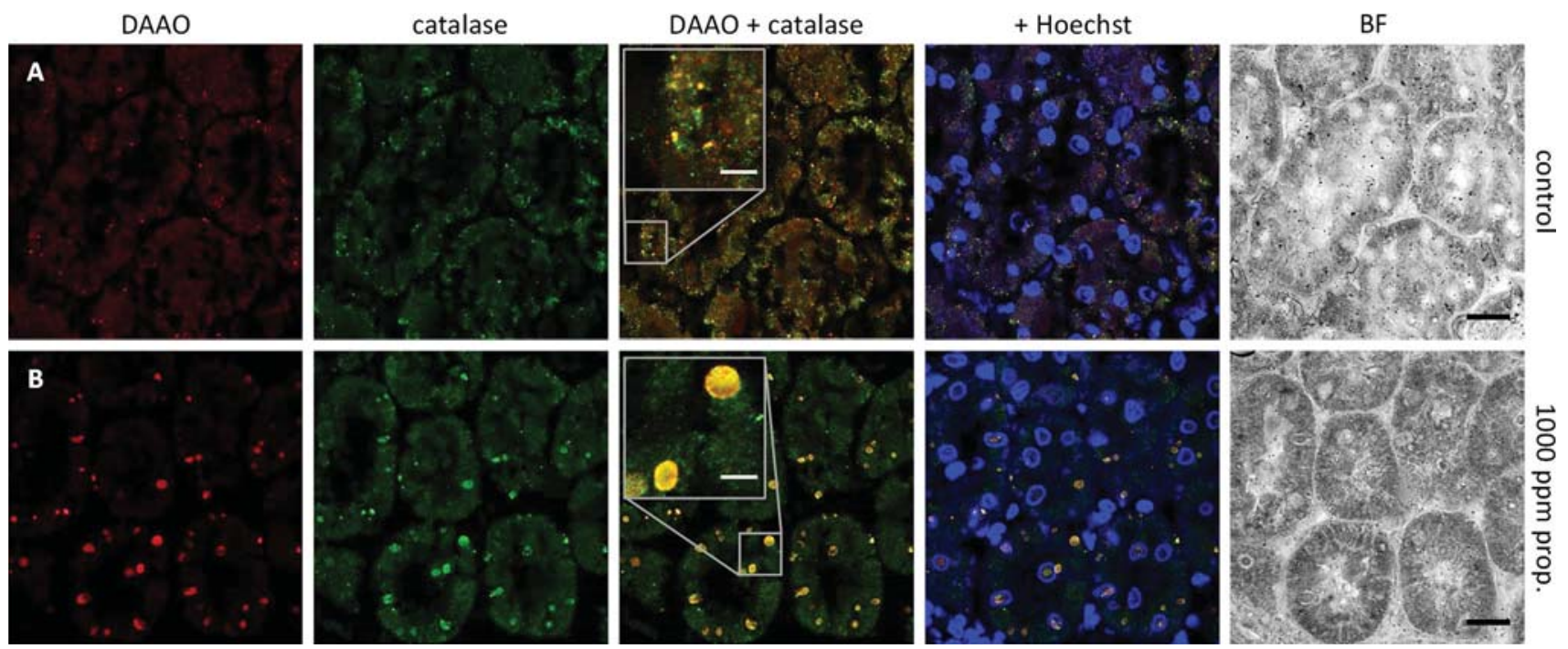

Fig. 4 Propiverine-induced changes in catalase localization. Confocal microscopy of kidney sections of control and propiverine-treated male F344 rats. Co-staining of rDAAO and peroxisomal enzyme catalase. Scale bar $20 \mu \mathrm{m}$, scale bar in magnification box: $5 \mu \mathrm{m}$. BF bright field, prop. propiverine
(I)

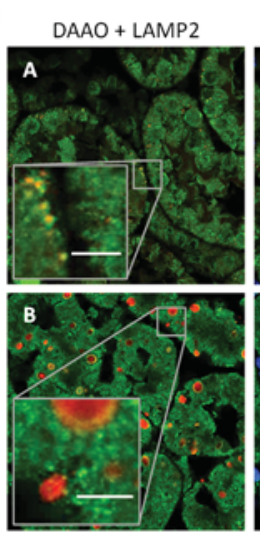

(IV)

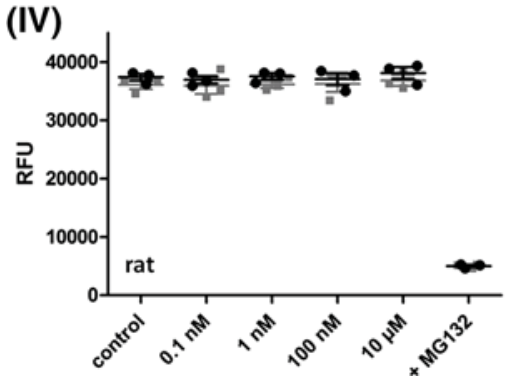

(II)
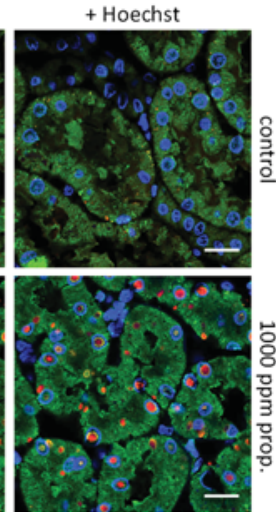

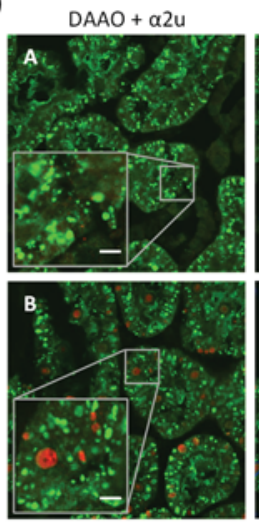

+ Hoechst
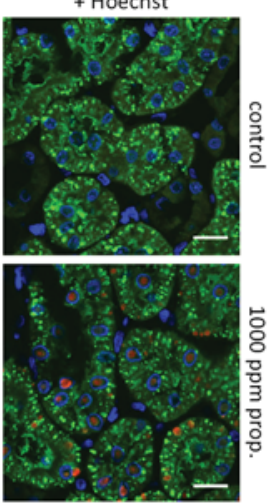

(III)
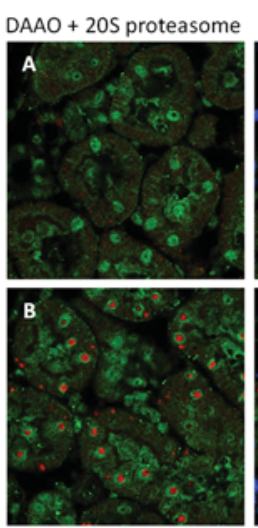

\section{.} .

. 
nuclei in proximal tubule cells of F344 rats (Fig. 5-III, costaining with Hoechst). Moreover, no change in the intensity of proteasomal staining upon propiverine treatment was observed, suggesting that proteasomal expression was not altered (Fig. 5-III, B). In addition, staining with an antibody against mono- and polyubiquitylated conjugates (FK2) pointed to ubiquitylation of proteins within the cytosolic and nuclear accumulations (data not shown). As rDAAO and $20 \mathrm{~S}$ proteasomes are both localized within nuclei of propiverine-treated rats (Fig. 5-III, B), we determined whether propiverine could inhibit proteasomal activity thereby resulting in the nuclear accumulation of peroxisomal proteins, e.g., rDAAO and catalase. Therefore, we performed a cell-free proteasome activity assay using fluorogenic peptide substrates of the caspase- and chymotrypsin-like activity of the 20S proteasome, respectively (Fig. 5-IV). The proteasomal inhibitor MG132 efficiently inhibited the caspase-like activity of the 20S proteasomes of rats, humans and mice (Fig. 5-IV). In contrast, neither propiverine nor its major metabolite propiverine- $N$-oxide decreased the proteasomal activity at any of the concentrations tested (Fig. 5IV). Comparable results were obtained using substrates for the chymotrypsin-like activity (data not shown). Taken together, we verified that rDAAO accumulations are not located in lysosomes. Moreover, we demonstrated that neither propiverine nor its major metabolite impaired proteasomal degradation by acting as proteasomal inhibitors.

\section{Discussion}

In general, protein accumulation in the proximal tubule can be caused by several reasons: First, the expression of the accumulating protein is increased (Tyedmers et al. 2010). Second, the expressed or reabsorbed protein is difficult to hydrolyze as estimated by a long half-life (Hard et al. 1993). Third, the catabolic capacity, consisting of lysosomal and proteasomal activity, is sufficiently reduced (Hard et al. 1993). In context with the latter, the apparently species-specific DAAO accumulation in the renal cortex of rats with the exposure to propiverine and other compounds (Gopinath et al. 1987; Hard and Snowden 1991; Dietrich et al. 2008; Radi et al. 2013; Shimoyama et al. 2014) is reminiscent of the well-known species- and sex-specific accumulation of $\alpha 2 \mathrm{u}$-globulin in rats, also causally related to the exposure to numerous chemicals (Swenberg 1993). Contrary to $\alpha 2 \mathrm{u}$-globulin, which is synthesized in the liver of male rats under androgenic control and reabsorbed in the proximal tubules (Borghoff et al. 1990), DAAO appears to be transcribed and translated in the proximal tubule proper (Konno et al. 1997). Treatment of rats with propiverine did not increase renal proximal tubule
rDAAO mRNA level (Heussner et al. 2016), thus excluding increased gene expression as a potential explanation of the propiverine-mediated rDAAO accumulation. However, both DAAO and $\alpha 2 \mathrm{u}$-globulin inherently have a long half-life (hDAAO: approx. $60 \mathrm{~h}$ (Cappelletti et al. 2013); $\alpha 2 \mathrm{u}$-globulin: approx. $6 \mathrm{~h}$ (Lehman-McKeeman et al. 1990)), whereby binding of chemicals to $\alpha 2 \mathrm{u}$-globulin extends this half-life markedly and exacerbates the accumulation of $\alpha 2 \mathrm{u}$-globulin in the lysosomes of exposed male rats (Swenberg et al. 1989; Lehman-McKeeman et al. 1990; Frazier et al. 2012). Consequently, we investigated whether propiverine interacts with DAAO in vitro, thus resulting in the cytosolic and nuclear DAAO accumulations observed in vivo. However, contrary to expectations, our results clearly demonstrated that propiverine and its major metabolic propiverine- $N$-oxide do not bind to rat or human DAAO or changed the tertiary structure of the enzyme, thus unlikely affecting DAAO stability. As the rDAAO amino acid sequence contains a bona fide and a putative chaperone-mediated autophagy (CMA)-targeting motif, as does a2u-globulin (Dice 1990; Cuervo et al. 1999), we determined whether propiverine treatment could affect lysosomal trafficking of renal proteins thus resulting in the mislocalization of $\alpha 2 \mathrm{u}$-globulin and/or rDAAO. However, we did not observe an overt redistribution of $\alpha 2 \mathrm{u}$-globulin nor a specific translocation of $\alpha 2 \mathrm{u}$-globulin into the nuclei of propiverine-treated rats, suggesting that propiverine did not affect the integration of $\alpha 2 \mathrm{u}$-globulin into lysosomes. Moreover, despite the presence of a CMAtargeting motif, we did not detect rDAAO in lysosomes of propiverine-treated rats, thus suggesting that propiverine does not affect lysosomal trafficking and that lysosomal degradation plays only a minor role in the catabolism of rDAAO. In contrast, we did observe a propiverine-mediated shift of rDAAO from a peroxisomal to a nuclear and cytosolic localization. The latter observation was coupled with a decreased mean peroxisomal size. Generally, peroxisomes are known to be highly variable in size, shape, number and protein content (Fransen 2012); nevertheless, to the best of our knowledge, a significant decrease in peroxisomal size after drug administration was not reported so far for mammalian cells. Interestingly, it is reported that peroxisomal proteins can be differentially localized within the cell depending on differential splicing, multiple targeting signals or phosphorylation (Fordor et al. 2012; Ast et al. 2013). However, dual localization of peroxisomal proteins was predominantly reported to occur into the cytosol and into mitochondria (Ast et al. 2013), merely one study in yeast described nuclear translocation of a peroxisomal NAD ${ }^{+}$-dependent glycerol 3-phosphate dehydrogenase (Jung et al. 2010). The concurrent analysis of the intracellular distribution of catalase, another peroxisomal protein, demonstrated a comparable propiverine-mediated 
mislocalization from the peroxisomes to the cytosol and nucleus, thereby suggesting the presence of more generalized compound-mediated reduced or abrogated translocation to or import of peroxisomal proteins into peroxisomes. It is important to note that catalase, similar to DAAO and $\alpha 2 \mathrm{u}$-globulin, is a protein with a long half-life (30 h) (Poole et al. 1969), thus lending support to the initial assumption that compound-induced changes in protein handling of long-lived proteins will lead to their prominent accumulation (Hard et al. 1993). The nearly double half-life of DAAO, when compared to catalase, may also explain why the accumulation of rDAAO in the cytosol and nuclei appeared more prominent than that of catalase.

The question, however, remains, why the peroxisomal rDAAO and catalase were found in the nuclei of propiverine-treated rats. Given the fact that neither DAAO nor catalase contains an intrinsic nuclear translocation signal and has a molecular size that renders passive diffusion through nuclear pores unlikely, we speculate that the accumulating proteins either entered the nucleus by piggybacking or by active import. The latter, however, would imply that these proteins are actively imported as a result of the inhibition of their original function and thus were marked for protein degradation. Indeed, recent studies provide compelling evidence that cytosolic misfolded proteins are actively imported into the nucleus for proteasomal degradation (Prasad et al. 2010; Park et al. 2013; Shibata and Morimoto 2014). Having found that the major fraction of 20 S proteasomes is located in nuclei of both placebo- and propiverinetreated F344 rats and that the BioGRID interaction database provides evidence that DAAO interacts with KLHL42, a substrate-specific adapter of an E3 ubiquitin-protein ligase, ${ }^{1}$ we wanted to demonstrate that proteins, e.g., DAAO and catalase are imported into the nucleus for proteasomal degradation. Co-staining with antibodies against mono- and polyubiquitylated conjugates indicated that cytosolic and nuclear protein accumulations are positive for ubiquitin (data not shown). This is in line with the fact that hDAAO was shown to be ubiquitylated in glioblastoma cells (Cappelletti et al. 2013). Concurrent manipulative in vitro experiments also demonstrated that proteasomal inhibition resulted in a comparable cytosolic and nuclear accumulation of DAAO (Luks et al., unpublished data). Based on the latter finding, we raised the question whether propiverine could not only affect peroxisomal protein trafficking, but also could act as a proteasomal inhibitor. The subsequent in vitro analyses employing rat, human and mouse 20S proteasome preparations, respectively, however, demonstrated that neither propiverine nor its major

\footnotetext{
${ }^{1}$ http://thebiogrid.org/107980, 20.11.15.
}

metabolite propiverine- $N$-oxide had any inhibitory effect on the $20 \mathrm{~S}$ proteasome.

In conclusion, we could rule out similarities between $\alpha 2 \mathrm{u}$-globulin droplet formation and rDAAO accumulation in rat kidney. In addition, we provide the first direct evidence that propiverine decreases peroxisomal size of rat proximal tubule cells. Co-staining of rDAAO and catalase revealed that the propiverine-induced protein accumulations do not consist of solely one protein and involve other peroxisomal proteins. These findings therefore strongly suggest that propiverine treatment affects the intracellular localization and/or peroxisomal trafficking of long-lived peroxisomal proteins such as rDAAO and catalase. Moreover, the fact that compounds other than propiverine were also shown to induce above protein accumulation may suggest that this describes a much broader phenomenon. Thus, elucidating the underlying mechanism of $\mathrm{rDAAO}$ accumulation will provide a better understanding of species-specific findings during non-clinical drug safety testing and will therefore result in an improved human health risk assessment. Indeed, since DAAO has been a pharmacological target (Sacchi et al. 2013), the suggested differences in propiverineinduced accumulation between rats and humans also raise questions regarding the appropriateness of rats as a model system for testing new drugs for the treatment of schizophrenia and neuropathic pain (Frattini et al. 2011).

Acknowledgments This work was supported by the Deutsche Deutsche Forschungsgemeinschaft, DFG (RTG 1331). LP and SS were supported by Grant from Fondo di Ateneo per la Ricerca. We gratefully acknowledge Apogepha Arzneimittel GmbH (Germany) for supplying propiverine and propiverine- $N$-oxide. We thank Dr. S. Erath and Prof. Dr. M. Groettrup for providing substances for, and hands-on instructions with, the proteasome activity assay as well as K. Collins and C. Grimm for technical assistance. In addition, we thank the BioImaging Center (BIC, University of Konstanz) for instrumentation.

Author contributions L. Luks designed and performed most of the experiments, analyzed, interpreted and discussed the evolving data, and wrote the manuscript. S. Sacchi and L. Pollegioni performed the in vitro binding experiments and edited the manuscript. D.R. Dietrich designed the overall research goals and approaches, interpreted and discussed the evolving data, and was deeply involved in manuscript writing. All authors read and approved the final manuscript.

\section{References}

Abrams P, Andersson K (2007) Muscarinic receptor antagonists for overactive bladder. BJU Int 100:987-1006. doi:10.1111/j.1464-410X.2007.07205.X

Ast J, Stiebler AC, Freitag J, Bölker M (2013) Dual targeting of peroxisomal proteins. Front Physiol 4:297. doi:10.3389/ fphys.2013.00297

Borghoff SJ, Short BG, Swenberg JA (1990) Biochemical mechanisms and pathobiology of alpha $2 \mathrm{u}$-globulin nephropathy. Annu Rev Pharmacol Toxicol 30:349-367. doi:10.1146/annurev. pa.30.040190.002025 
Caldinelli L, Iametti S, Barbiroli A et al (2005) Dissecting the structural determinants of the stability of cholesterol oxidase containing covalently bound flavin. J Biol Chem 280:22572-22581. doi:10.1074/jbc.M500549200

Caldinelli L, Molla G, Bracci L et al (2010) Effect of ligand binding on human D-amino acid oxidase: implications for the development of new drugs for schizophrenia treatment. Protein Sci 19:1500-1512. doi:10.1002/pro.429

Cappelletti P, Campomenosi P, Pollegioni L, Sacchi S (2013) The degradation (by distinct pathways) of human D-amino acid oxidase and its interacting partner pLG72 - two key proteins in D-serine catabolism in the brain. FEBS J. doi:10.1111/febs.12616

Cuervo AM, Hildebrand H, Bomhard EM, Dice JF (1999) Direct lysosomal uptake of alpha 2-microglobulin contributes to chemically induced nephropathy. Kidney Int 55:529-545. doi:10.1046/j.1523-1755.1999.00268.x

Dice J (1990) Peptide sequences that target cytosolic proteins for lysosomal proteolysis. Trends Biochem Sci 15:305-309

Dietrich DR, Rasonyi T (1995) Preneoplastic lesions in kidney and carcinogenesis by non-genotoxic compounds. Arch Toxicol Suppl 17:536-546. doi:10.1016/0378-4274(94)90255-0

Dietrich DR, Heussner AH, O'Brien E et al (2008) Propiverineinduced accumulation of nuclear and cytosolic protein in F344 rat kidneys: isolation and identification of the accumulating protein. Toxicol Appl Pharmacol 233:411-419. doi:10.1016/j. taap.2008.09.014

Erath S, Groettrup M (2014) No evidence for immunoproteasomes in chicken lymphoid organs and activated lymphocytes. Immunogenetics 67:51-60. doi:10.1007/s00251-014-0814-1

Fordor K, Wolf J, Erdmann R et al (2012) Molecular requirements for peroxisomal targeting of alanine-glyoxylate aminotransferase as an essential determinant in primary hyperoxaluria type 1 . PLoS Biol. doi:10.1371/journal.pbio.1001309

Fransen M (2012) Peroxisome dynamics: molecular players, mechanisms, and (dys)functions. ISRN Cell Biol 2012:1-24. doi: $10.5402 / 2012 / 714192$

Frattini LF, Piubelli L, Sacchi S et al (2011) Is rat an appropriate animal model to study the involvement of D-serine catabolism in schizophrenia? Insights from characterization of D-amino acid oxidase. FEBS J 278:4362-4373. doi:10.1111/j.1742-4658.2011.08354.x

Frazier KS, Seely JC, Hard GC et al (2012) Proliferative and nonproliferative lesions of the rat and mouse urinary system. Toxicol Pathol 40:14S. doi:10.1177/0192623312438736

Gopinath C, Prentice DE, Lewis DJ (1987) Atlas of experimental toxicological pathology. Springer, Netherlands

Hard GC, Snowden RT (1991) Hyaline droplet accumulation in rodent kidney proximal tubules: an association with histiocytic sarcoma. Toxicol Pathol 19:88-97. doi:10.1177/019262339101900202

Hard GC, Rodgers IS, Baetcke KP et al (1993) Hazard evaluation of chemicals that cause accumulation of alpha( $2 u)$-globulin, hyaline droplet nephropathy, and tubule neoplasia in the kidneys of male rats. Environ Health Perspect 99:313-349

Hard G, Alden C, Bruner R, Frith C (1999) Non-proliferative lesions of the kidney and lower urinary tract in rats. Guides for Toxicologic Pathology, STP/ARP/AFIP, Washington, DC

Harris CM, Molla G, Pilone MS, Pollegioni L (1999) Studies on the reaction mechanism of Rhodotorula gracilis D-amino-acid oxidase. Role of the highly conserved Tyr-223 on substrate binding and catalysis. J Biol Chem 274:36233-36240. doi:10.1074/ jbc. 274.51 .36233

Haustein K, Huller G (1988) On the pharmacokinetics and metabolism of propiverine in man. Eur J Drug Metab Pharmacokinet 13:81-90

Heussner AHH, Sprinz MI, Balaguer P, Dietrich DR (2016) Probing the PPAR involvement in the propiverine-induced renal DAAO accumulation in rats. PPAR Res (Submitted)
Inoue H, Enomoto M, Kobayashi K (1989) Two-year oral carcinogenicity study in mice (B6C3F1) with $\mathrm{P} 4$ (propiverine hydrochloride). Rep No 1556 (Apogepha ID P150) p 258

Iwata A, Nagashima Y, Matsumoto L et al (2009) Intranuclear degradation of polyglutamine aggregates by the ubiquitin-proteasome system. J Biol Chem 284:9796-9803. doi:10.1074/jbc. M809739200

Jung S, Marelli M, Rachubinski RA et al (2010) Dynamic changes in the subcellular distribution of Gpd1p in response to cell stress. J Biol Chem 285:6739-6749. doi:10.1074/jbc.M109.058552

Kohda S, Nishikawa H, Sumino M et al (1989) One-year chronic oral toxicity study of propiverine hydrochloride in dogs followed by one-month recovery. J Toxicol Sci 14:111-160

Konno R (1998) Rat D-amino-acid oxidase cDNA: rat D-aminoacid oxidase as an intermediate form between mouse and other mammalian D-amino-acid oxidases. Biochim Biophys Acta 1395:165-170

Konno R, Sasaki M, Asakura S et al (1997) D-Amino-acid oxidase is not present in the mouse liver. Biochim Biophys Acta 1335:173-181

Konno R, Okamura T, Kasai N et al (2009) Mutant rat strain lacking D-amino-acid oxidase. Amino Acids 37:367-375. doi:10.1007/ s00726-008-0163-1

Latonen L (2011) Nucleolar aggresomes as counterparts of cytoplasmic aggresomes in proteotoxic stress. Bioessays 33:386-395. doi:10.1002/bies.201100008

Latonen L, Moore HM, Bai B et al (2011) Proteasome inhibitors induce nucleolar aggregation of proteasome target proteins and polyadenylated RNA by altering ubiquitin availability. Oncogene 30:790-805. doi:10.1038/onc.2010.469

Lee DH, Goldberg AL (1998) Proteasome inhibitors:valuable new tools for cell biologists. Trends Cell Biol 8:397-403

Lehman-McKeeman LD, Rivera-Torres MI, Caudill D (1990) Lysosomal degradation of $\alpha 2 \mathrm{u}$-globulin and $\alpha 2 \mathrm{u}$-globulin-xenobiotic conjugates. Toxicol Appl Pharmacol 548:539-548

Madersbacher H, Mürtz G (2001) Efficacy, tolerability and safety profile of propiverine in the treatment of the overactive bladder (non-neurogenic and neurogenic). World J Urol 19:324-335

May K, Westphal K, Giessmann T et al (2008) Disposition and antimuscarinic effects of the urinary bladder spasmolytics propiverine: influence of dosage forms and circadian-time rhythms. J Clin Pharmacol 48:570-579. doi:10.1177/0091270008315314

Molla G, Sacchi S, Bernasconi M et al (2006) Characterization of human D-amino acid oxidase. FEBS Lett 580:2358-2364. doi:10.1016/j.febslet.2006.03.045

Nakano S, Kuwata M, Hasegawa H et al (1989) Thirteen-week oral toxicity study of propiverine hydrochloride in rats. J Toxicol Sci. doi:10.2131/jts.14.SupplementII_13

Park S-H, Kukushkin Y, Gupta R et al (2013) PolyQ proteins interfere with nuclear degradation of cytosolic proteins by sequestering the Sis1p chaperone. Cell 154:134-145. doi:10.1016/j. cell.2013.06.003

Pilone MS (2000) D-Amino acid oxidase: new findings. Cell Mol Life Sci 57:1732-1747

Pollegioni L, Piubelli L, Sacchi S et al (2007) Physiological functions of D-amino acid oxidases: from yeast to humans. Cell Mol Life Sci C 64:1373-1394. doi:10.1007/s00018-007-6558-4

Poole B, Leighton F, De Duve C (1969) The synthesis and turnover of rat liver peroxisomes. Turnover of peroxisome proteins. J Cell Biol 41:536-546. doi:10.1083/jcb.59.2.507

Prasad R, Kawaguchi S, Ng DTW (2010) A nucleus-based quality control mechanism for cytosolic proteins. Mol Biol Cell 21:2117-2127. doi:10.1091/mbc.E10

Radi ZA, Stewart ZS, Grzemski FA, Bobrowski WF (2013) Renal pathophysiologic role of cortical tubular inclusion bodies. Toxicol Pathol 41:32-37. doi:10.1177/0192623312450629 
Read NG (1991) The role of lysosomes in hyaline droplet nephropathy induced by a variety of pharmacological agents in the male rat. Histochem J 23:436-443

Reits EA, Benham AM, Plougastel B et al (1997) Dynamics of proteasome distribution in living cells. EMBO J 16:6087-6094. doi:10.1093/emboj/16.20.6087

Sacchi S, Caldinelli L, Cappelletti P et al (2012) Structure-function relationships in human D-amino acid oxidase. Amino Acids 43:1833-1850. doi:10.1007/s00726-012-1345-4

Sacchi S, Rosini E, Pollegioni L, Molla G (2013) D-Amino acid oxidase inhibitors as a novel class of drugs for schizophrenia therapy. Curr Pharm Des 19:2499-2511. doi: $10.2174 / 1381612811319140002$

Shibata Y, Morimoto RI (2014) How the nucleus copes with proteotoxic stress. Curr Biol 24:R463-R474. doi:10.1016/j. cub.2014.03.033

Shimoyama N, Nakatsuji S, Andoh R et al (2014) Spontaneously occurring formation of intranuclear and cytoplasmic inclusions in renal proximal epithelium due to accumulation of D-amino acid oxidase in Wistar Hannover rats. Toxicol Pathol. doi: $10.1177 / 0192623314560611$

Subak L, Richter H, Hunskaar S (2009) Obesity and urinary incontinence: epidemiology and clinical research update. J Urol 182:35. doi:10.1016/j.juro.2009.08.071.Obesity

Sun Y, Yu H, Zheng D et al (2011) Sudan black B reduces autofluorescence in murine renal tissue. Arch Pathol Lab Med 135:13351342. doi:10.5858/arpa.2010-0549-OA

Swenberg JA (1993) Alpha 2u-globulin nephropathy: review of the cellular and molecular mechanisms involved and their implications for human risk assessment. Environ Health Perspect 101(Suppl 6):39-44
Swenberg J, Short B, Borghoff S (1989) The comparative pathobiology of $\alpha 2 \mathrm{u}$-globulin nephropathy. Toxicol Appl Pharmacol 46:35-46. doi:10.1016/0041-008X(89)90053-7

Tyedmers J, Mogk A, Bukau B (2010) Cellular strategies for controlling protein aggregation. Nat Rev Mol Cell Biol 11:777-788. doi: $10.1038 / \mathrm{nrm} 2993$

Usuda N, Yokota S, Hashimoto T, Nagata T (1986) Immunocytochemical localization of D-amino acid oxidase in the central clear matrix of rat kidney peroxisomes. J Histochem Cytochem 34:1709-1718. doi:10.1177/34.12.2878022

von Mikecz A (2006) The nuclear ubiquitin-proteasome system. J Cell Sci 119:1977-1984. doi:10.1242/jcs.03008

Wein A (2011) Symptom-based diagnosis of overactive bladder: an overview. Can Urol Assoc J 5:S135-S136. doi:10.5489/ cuaj. 11183

Wójcik C, DeMartino GN (2003) Intracellular localization of proteasomes. Int J Biochem Cell Biol 35:579-589. doi:10.1016/ S1357-2725(02)00380-1

Wuest M, Weiss A, Waelbroeck M et al (2006) Propiverine and metabolites: differences in binding to muscarinic receptors and in functional models of detrusor contraction. Naunyn Schmiedebergs Arch Pharmacol 374:87-97. doi:10.1007/s00210-006-0103-0

Yamada S, Ito Y, Taki Y et al (2010) The N-oxide metabolite contributes to bladder selectivity resulting from oral propiverine: muscarinic receptor binding and pharmacokinetics. Drug Metab Dispos 38:1314-1321. doi:10.1124/dmd.110.033233

Yamashita K, Nakano S, Kuwata M et al (1989) Acute toxicity studies of propiverine hydrochloride. J Toxicol Sci 14:1-11

Yamashita K, Kuwata M, Irimura K et al (1990) Fifty-two-week oral chronic toxicity study of propiverine hydrochloride in rats. J Toxicol Sci 15:107-144. doi:10.2131/jts.15.107 